\title{
A review on various methods of collaborative computing
}

\author{
Mohd Afiq Bin Zamanhuri ${ }^{1}$, Zalilah Abd Aziz $^{2}$, Rose Hafsah Abd Rauf ${ }^{3}$, \\ Elly Johana Johan ${ }^{4}$, Noratikah Shamsudin ${ }^{5}$ \\ ${ }^{1,2,3,5}$ Faculty of Computer and Mathematical Sciences, Universiti Teknologi MARA, Malaysia \\ ${ }^{4}$ Department of Computer Science and Mathematics, Universiti Teknologi MARA, Malaysia
}

\begin{abstract}
Article Info
Article history:

Received Dec 20, 2018

Revised Mar 17, 2019

Accepted Apr 11, 2019

\section{Keywords:}

Centralized computing

Collaborative computing

Distributed computing

Islamic lectures online

Web-based
\end{abstract}

\section{Corresponding Author:}

Mohd Afiq Bin Zamanhuri,

Faculty of Computer and Mathematical Sciences,

Universiti Teknologi MARA,

40450 Shah Alam, Selangor, Malaysia.

Email: afiqzamanhuri@gmail.com

\begin{abstract}
Currently, mosques in Malaysia distribute their lecture schedules either on paper-based form or by uploading the schedule on their social media platform. This has some disadvantages such as paper schedules are susceptible to damages and information on social media platform is often not updated to current changes. Collaborative Computing is a system that enable individuals to work together remotely by making use of the reach ability of the internet. In order to utilise the Internet's obvious advantages over paper-based and rapid information distribution and asynchronous communication, a review is conducted to study the available methods of collaborative computing, further analyse current research papers. Result shows that Centralized Computing method is the most suitable method for developing collaborative mobile application for Islamic Lectures schedule.
\end{abstract}

Copyright (c) 2019 Institute of Advanced Engineering and Science. All rights reserved.

\section{INTRODUCTION}

Seeking knowledge is a duty upon every Muslims. The advancement of Information and Communication Technology (ICT) have evolved most of our daily routine[1]. For example, the book of Allah, the Al Quran, can be find in numerous mobile application. Those applications also included the audio of the recitations with the translations of each word. Today, the internet has been the main medium to spread the teachings of Islam [2]. Most of the application took the asynchronous approach, even though the use of ICT offers strong learning settings and able to alter the learning and teaching process in an operative, self-directed way [1]. As discovered by Martin, Parker \& Allred [3], some of the disadvantages of asynchronous virtual learning are less social interaction, the lack of immediate feedback, and students feel isolated. Thus, for learning Islamic knowledge, it is more appropriate to attend the physical lectures at the mosques. In Malaysia, apart from searching for the videos of Islamic lectures online, the people are still practicing the traditional way, which are go to the mosques, to listen to the lectures. Each mosque has their own paper-based schedule which is produced on monthly basis. The schedules are mostly placed at various places inside the mosque. The audiences depend on those schedules to know the information about the lectures. The problem with this method is the dissemination of the information. The audience may not notice the spreading of the schedule and the paper schedules are susceptible to damages. The schedule also may not be available to all audience because the audience may outnumber the quantity of the printed schedule. A paper-based schedule does not possess the ability of easy access to the internet. Furthermore, the traditional way has lack of notification function. There is no telling the mosque goers that the lecture has been cancelled. Besides that, there is no central hub that enables the user to access the schedule. The importance of having a one-stop access was verified by using social networking tools. Another example is a computer-based testing via the Internet shows that the internet allows a more diverse sample to be located 
as the participant can take the test at home without the need to go to the actual place of the test [4]. It is also reported that the internet can offer library resources including answer enquiries, catalogue search and information about new collections and lists, to convey general library information, and to offer online resources [5]. Lastly, the existing applications are not efficient. They are either not user-friendly or not supported anymore by the developer. The schedules are displayed in the form of low-resolution images [4].

The Islamic lectures are a kind of education bestow upon an opportunity for the audience to contemplate different answers to major religious and moral issues to direct them in developing their interpretation in a reflective way [2]. Because of that, the schedules need to be spread in a better way, so that the audience can keep up-to-date with the lectures. Thus, this project proposed a solution to all the problems stated, which is a mobile apps schedule organizer for Islamic Lectures. It is a mobile application because almost everyone has their own mobile device. It works similar to the existing application, but the schedule of each mosque is handled by each mosque's committee. Any mosque can put in their schedule into the system, and updating it later on. The system also has identification security. This decision is made in order to avoid any misconduct on producing any misleading schedule. This application will utilise the Internet's obvious advantages over paper-based such as rapid information distribution and asynchronous communication. As stated in $[1,6]$, those abilities are vital for a large community, where the sheer number of people involved makes face-to-face meetings costly and difficult to organise.

This paper aims to fulfil three objectives, one of which is to study about Collaborative Computing and identify its various methods available. Collaborative Computing is a system that enable individuals to work together remotely by making use of the reach ability of internet [7]. Secondly is to analyse and make a review between the available methods and lastly to evaluate the most suitable method to apply for the project. This project aims to develop a one-stop mosque's lecture schedule application which involve two kind of users; first the public user which can only view the updated schedule and secondly, the administrator, which are the mosques' committee who can create, update and modify the schedule. Furthermore, this application can act as a one-stop information hub about the schedule of Islamic lectures. Besides, it can also provide reminders to community about the lectures available. Finally, the users will be able to view the schedule of desired mosque on that current month. Thus, before development phase, the next section will be discussed in depth on the literature review on system's architecture, to better understand its structure.

\section{LITERATURE REVIEW}

As explained in previous section, the proposed solution differs from the existing application by involving the mosque's committee to input their schedule into the system. This collaboration act is the core principle of the system and this led to the selection of Collaborative Computing to be the mainframe for this system. Table 1 shows the existing applications characteristics.

Table 1. Characteristics of Existing Applications

\begin{tabular}{|c|c|c|c|}
\hline Criteria Apps & Recent Update & Function for Schedule Update & User Interface and navigation \\
\hline Jadual Program Agama & 2014 & $\begin{array}{l}\text { Yes, but any user can update any } \\
\text { mosques schedule }\end{array}$ & $\begin{array}{ll}- & \text { Minimalist approach } \\
\text { - } & \text { Have a "Search" function. }\end{array}$ \\
\hline Kuliah Agama & 2014 & $\begin{array}{l}\text { Yes, but the developer needs to } \\
\text { update it }\end{array}$ & $\begin{array}{l}\text { - } \\
\text { - Have a category of schedules (mosque or } \\
\text { lecturer) but without "Search" function. }\end{array}$ \\
\hline Majlis Ilmu Pulau Pinang & 2017 & $\begin{array}{l}\text { Yes, but the developer needs to } \\
\text { update it }\end{array}$ & $\begin{array}{l}\text { - Tedious scroll is needed to find the desired } \\
\text { schedule. } \\
\text { - Have a category of schedules (monthly or } \\
\text { daily) but without "Search" function. }\end{array}$ \\
\hline
\end{tabular}

By definition, Collaborative Computing is essentially a system that enabling individuals to work together remotely by making use of the reach ability of internet [7]. Collaborative computing can connect individuals to software applications in real time, so they all can access and simultaneously work on text-based documents, graphics, computer-aided design files and other work products. Thus, this concept is suitable for the authors' project, as the mosque committee themselves will create the monthly schedule and upload it into the application. The prime examples of the early Collaborative Computing are online video and audio conferencing. Previously, this technology aimed to bridge communications that is physically limited. Since then, Collaborative Computing has evolved that introduced real-time environment which enables documents to be edited simultaneously in a workgroup. Nowadays, cloud computing also features Collaborative Computing service. Among its benefits, it provides a collaborative environment workgroup computing, optimizes productivity, and fosters innovation. Previously, Collaborative System co-dependent to telecommunications network as the primary communication medium. Since then, they can be used by devices 
that connected to local area networks (LANs). Beyond these characteristics, Collaborative Computing can be divided into two categories based on their operation which are Centralized Computing and Distributed Computing. In the next two sections these categories will be explained in detail.

\subsection{Centralized Computing}

In this method, the architecture's processing is being done in all or mostly in the central device. The method is very much alike to the client/server architecture where the central device is connected to a number of remote devices. The central server is deployed as the main application or hub that has massive computing resources, storage and other additional features. As the foundation of Collaborative Computing architecture, one or more remote devices are directly connected to a main device. In turn, the main device is responsible for delivering application's logic, processing and providing computing resources to the attached devices. Because of this, the administrator in a centralized computing infrastructure will manage all the connected remote machines from the central machine interface. Apart from the obvious features that can be seen directly from the method's characteristics, the other features of Centralized Computing are as follows:

- As all the remote devices are connected to the main device, thus, all means and resources are obtainable.

- The new connections between the computing system's office locations and the data centre are normally of low capacity, short delay or uncertain.

- The programming language used is the same throughout the development phase.

A Centralized Computing system has several advantages. One of such is that the database can be easily managed as all of the data about the schedule will be located in single repository. The data stored is easier to be changed, re-organized, mirrored, or analysed and the data will be a lot secure. Furthermore, the data redundancy will be at minimum because as researched by [8] as there will be no additional registries of the same patients need to be built to ensure the compatibility of Red Blood Cell unit of that patients' as long as they are transfused only at the hospitals that have access to the same database. Other advantage is Centralized computing processing reduces the expenditure as it does not point up on additional machines and hardware. As the main devices doing most of the processing, the disadvantages will be from that main device. Firstly, it demands a large data storage, as the main device will usually create and store the data. Besides that, the central machine will be an easy target for malicious threats and it is not suitable for the use of different hardware and vendors. Furthermore, the devices' ability to response is reduced to the information request timely. Lastly, the system will require a high cost in transmitting transactions.

\subsection{Distributed Computing}

Distributed computing is a field of computer science that studies distributed systems, a system whose components are located on different networked computers, which then communicate and coordinate their actions by passing messages to one another. In this architecture, information processing is not confined to a single device rather it is distributed over several independent computers, thus, the associated devices shared tasks as well as program components. This task-sharing method can improve the overall system's efficiency and performance as any modification can be done without affecting the overall system [9]. Distributed system consists of middleware that manage or support the different components of a distributed system. It provides a buffer between the applications and the network. Figure 1 shows the general Distributed Computing infrastructure.

The basis of a distributed computing is its transparency, accessibility and scalability. For transparency, it is important for a distributed system to hide the location of its process and resource. Besides that, is an important goal of distributed system in which it be accessible of using hardware and software from different vendors. Furthermore, scalability of the system is measured along three different dimensions. First, a system can be scalable with respect to its size which can add more user and resources to a system. Second, users and resources can be geographically apart. Third, it is possible to manage even if many administrative organizations are spanned. As proven by [10], in a distributed computing framework, able to employed the Minimum Bandwidth Code and achieving a scalable architecture with a constant load of communication.

One of the advantages of Distributed Computing is allows the processor to be used to share processing when not being used for other activities. The data used locally can be stored locally and network traffic kept to a minimum and if the data is lost on central site, it can be reduplicated from local site. Distributed system also able to continue its operation even after an error has occurred. On the flip side, in term of complexity, a Centralized system is simpler than Distributed system. Other than that, it is more susceptible to external attack, as all the data is not stored in one location if a local site does not have location adequate backup. It also has a higher overall cost than Centralized system. Lastly, the system's data is prone to inconsistency. In the next section of this paper, there is an analysis on the current research that has been done for these two methods. 


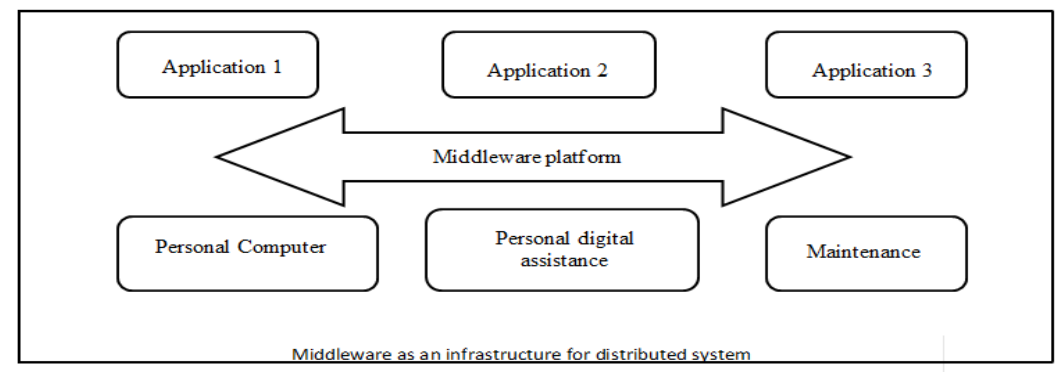

Figure 1. Distributed computing infrastructure [11]

\section{ANALYSIS}

The purpose for this analysis is to weigh-in the features for both Centralized Computing and Distributed Computing and selecting which is the most suitable to be applied to the system. A study of requirement for software development as in $[12,13]$ are also analyzed. The analysis involved eight current research papers and their results were evaluated. To help with the decisions, the researches were analysed and evaluated for the outcome.

\subsection{Peaynotes: A Generic Web-Based Patient Clinical Notes Sharing System for Health Care Professionals}

This research has been focusing on advancing the current note-taking method. The main problem is the inefficiency of current practice which the nurses need to wrote down notes then turns it to digital notes $[14,15]$. They also noticed the high cost of the technologically-advanced simulation system which hinder the hospital into taking such route. A proposed collaborative mobile application, called Peaynotes, replacing the current method. It is a modifiable bedside reporting tool and documentation of care system to be utilized during clinical simulation experiences. One of the features stated in that research is that the application has ability to retrieve valuable patient information to aid in effective communication during hand-off. It was developed using WordPress and follows the Centralized Computing method.

\subsection{Drop Computing: Ad-Hoc Dynamic Collaborative Computing}

In complete contrast from the research above, this paper used the cloud computing for eLearning collaboration system. The main issue that make the researcher chose cloud computing is that the traditional web-based e-learning mode models lack the underlying infrastructure that are highly scalable and can dynamically allocate the required computation and storage resources. Besides, the building and maintenance are located onsite which causes a lot of problems for institutions. Thus, they had proposed Cloud-Collaborative E-Learning Architecture that consists of 5 layers [16]:

- Infrastructure layer

- Software resource layer

- Resource management layer

- $\quad$ Service layer

- Application layer

Based from the paper, cloud computing has several security benefits, such as improved improbability and easy data monitoring. Cloud computing also invulnerable to system crashes since critical applications and data are stored in the cloud.

\subsection{Developing Distributed Computing Applications with Tasklets}

In this paper, it is not the whole system is proposed, instead a middleware of distributed computing system was proposed, in a form of Tasklets. The tasklet system allows developers to offload self-contained units of computation to a pool of heterogeneous computing devices. These tasklets approach was proposed is because of the increasing demand for computational performance of pervasive applications. It is a lightweight middleware thus, not affecting the performance of the connected devices. Once the Tasklet was executed and are connected to a broker they can contribute their computation power in form of virtual machines and run applications that offload computation via Tasklets [17].

\subsection{An Energy-Optimal Scheduling for Collaborative Execution in Mobile Cloud Computing}

For mobile application, the resources are very much limited for any serious processing. Thus, this research investigated the possible route for collaborative computing. Besides of resource limits, the other problem stated in the paper is Information such as the communication channels or the computation workload 
may change in different conditions because of the various versions of mobile devices. [18]. The researchers turned to the centralized-method cloud computing for the solution, which producing energy-optimal scheduling policy for collaborative execution in mobile cloud computing network. This policy has low-complexity threshold adaptation scheme for dynamical constrains of execution time in mobile cloud computing.

\subsection{Trustworthy Distributed Computing on Social Networks}

Different from the previous researches, this study focused on the huge network of social network. The researchers highlight the flaws cloud computing such as the need for architectures to support various potential applications, programming models to address large scale datacentric computing, new applications that benefit from the architectural and programming models in the cloud, and the need for strong security and data privacy protection guarantees [19]. Thus, a solution that is called SocialCloud was proposed. It is a distributed computing service that recruits computing workers from friends in social networks and use such social networks that characterize trust relationships to bootstrap trust in the proposed computing service.

\subsection{Task-Technology Fit and User Adoption of Cloudbased Collaborative Learning Technologies}

In this research, they study the acceptance of cloud-based collaborative learning technologies and gauges it using Task-Technology Fit (TTF) model to find factors that play a significant role. Their problem statements are the traditional teaching learning methods are not sufficient to support the expectations of academics and students in universities and there are no studies have looked at cloud usage and acceptance in a university setting [20]. The results are cloud applications with more personalized, mobility, and collaboration characteristics are more appropriate to support collaborative learning. In addition, non-routine tasks have negative influence on Task-Technology Fit. As the papers above have given a series of insight, the next subsection will discuss on the other disadvantages of both methods.

\subsection{Other Issue on Collaborative Computing}

After pointing out all the important points in those papers, it should be noted that there are several other issues that need to be addressed [21]. One of it is that the Centralized computing may have issues similar to client/server architecture because both look the same in structural sense [22]. Another issue is to consider the attributes that can contribute to algorithmic problem solving cempetencies [23]. Because of the complexity of Distributed Computing, enforcing a good security is equally complex as founded by [24] the system may require multi-level resource isolation to prevent inter-cluster access to network traffic and [25] cryptographic entropy is needed to secure the communications. After reviewing all the researches and issues of the two methods of Collaborative Computing, the characteristics of those methods are summarized in Table 2.

Table 2. The Characteristics of Centralized Computing Method

\begin{tabular}{ccc}
\hline Criteria & Centralized Computing & Distributed Computing \\
\hline Overall Cost & Lower & Higher \\
Vulnerability to threats & Lower & Higher \\
Complexity & Simpler & Complex \\
Storage Needed & Bigger & Smaller \\
Transparency & None & Obscure \\
Scalability & Poor & Good \\
Accessibility & Low & High \\
\hline
\end{tabular}

From above table, each of the method have its pros and cons, so, this can be used as a benchmark in selecting which methods works best for the project. The last section of this paper will discuss on the result of the analysis and future works of this project.

\section{CONCLUSION AND FUTURE WORKS}

After considering all the key points from the research papers, and by refereeing to the criteria from Table 2, the Centralized Computing method proved to be the best alternative. There are several reasons to support this selection. First of all is the lower overall cost as the centralized system cost will focus solely on the main machine. The cost also can be flowed to the storage as it needs a larger capacity of storage. Secondly, the system will be more secure even though the main device will be the only target. While the data backup plan is not as robust as the Distributed Computing system, but by having a good programming, this is makes data easy to secure and monitor. Lastly, while the chosen method has low hardware accessibility, 
but with Internet, the gap can be bridged as the proposed system is a web-based system. For future works, the project will proceed to its development phase to create a prototype of the system. The prototype will be focused on the main functionality of the system, which is the creation of the schedule and the display of the schedule.

\section{ACKNOWLEDGEMENTS}

Funding for this research was provided by Lestari Grant (0129/2016), Universiti Teknologi Mara.

\section{REFERENCES}

[1] Hosseini, S. E., Ramchahi, A. A., \& Raja Yusuf, R. J., "The impact of Information Technology on Islamic Behaviour," Journal of Multidisciplinary Engineering Science and Technology (JMEST), vol.1 Issue 5, pp. 135141, Available from: https://doi.org/10.13140/RG.2.1.4799.9603, 2014.

[2] Schreiner, P., "Religious Education in the European Context," 2013, Available from: https://doi.org/10.14413/HERJ2013.04.01,2013

[3] Martin, F., Parker, M., \& Allred, B., "A Case Study on the Adoption and use of Synchronous Virtual Classrooms," Electronic Journal of E-Learning, vol. 11 Issue 2, pp.124-138, 2013.

[4] Noyes, J. M., \& Garland, K. J., "Computer- vs. Paper-based Tasks: Are they equivalent?," Ergonomics, vol. 51 Issue 9, pp. 1352-1375, Available from: https://doi.org/10.1080/00140130802170387, 2008

[5] Hasan, M., "Use of Social Networking Sites in Library and Information Centres,", Available from: https://www.researchgate.net/profile/Aslam_Ansari4/publication/296241236_Use_of_Social_Networking_Sites_in _Library_and_Information_Centres/links/5706001108ae74a08e27539d.pdf, 2015

[6] Whittaker, S., \& Schwarz, H., "Meetings of the Board: The Impact of Scheduling Medium on Long Term Group Coordination in Software Development," Computer Supported Cooperative Work: CSCW: An International Journal, vol.8 Issue 3, pp. 175-205, Available from: https://doi.org/10.1023/A:1008603001894_ 1999.

[7] Kish J.S. (1995). Collaborative Computing. Advances in Parallel Computing. Vol (10), 351-357, 1995.

[8] Harm, S. K., Yazer, M. H., Monis, G. F., Triulzi, D. J., AuBuchon, J. P., \& Delaney, M., “A Centralized Recipient Database Enhances the Serologic Safety of RBC Transfusions for Patients With Sickle Cell Disease," American Journal of Clinical Pathology, vol, 141 Issue 2, pp. 256-261, Available from: https://doi.org/10.1309/AJCP47QAAXTOZEKJ. 2014.

[9] Ims, S. D., Li, Y., Lusardi, R. J., \& Xing, J., "U.S. Patent No. 9,137,324” Washington, DC: U.S. Patent and Trademark Office, 2015.

[10] Li, S., Maddah-Ali, M. A., \& Avestimehr, A. S., “A Unified Coding Framework for Distributed Computing with Straggling Servers," arXiv preprint arXiv:1609.01690, 2016.

[11] Tutorialspoint.com, "Software Architecture and Design Distributed Architecture.", Available from: https://www.tutorialspoint.com/software_architecture_design/distributed_architecture.htm.. (n.d).

[12] Mokhtar S.O., Nordin R., Aziz Z.A. \& Rawi R.M. Issues and Challenges of Requirements Review in the Industry. Indian Journal of Science and Technology. vol, 10 Issue 3, 2017.

[13] Mokhtar S. O., Nordin R., Aziz Z.A. \& Rawi R.M. Software Requirements Review Framework: A Case Study of Resource Tracking Project. International Journal of Control Theory and Applications. vol, 10 Issue 29, pp. 389-395, 2017.

[14] Church, J. C., Shank, B., Lee, T. S., Compton, T., \& Conklin, J. M., "Peaynotes: A generic Web-based Patient Clinical Notes Sharing System for Health Care Professionals," Journal of Computing Sciences in Colleges, vol, 34 Issue 2, pp. 58-64, 2018.

[15] Ciobanu, R. I., Negru, C., Pop, F., Dobre, C., Mavromoustakis, C. X., \& Mastorakis, G., "Drop Computing: Ad-hoc Dynamic Collaborative Computing," Future Generation Computer Systems, 2017.

[16] Naik, N. V., \& Madhavi, K., "Cloud Computing Architecture for Collaborative E-learning System," Applied and Theoretical Computing and Communication Technology (iCATccT), 2015 International Conference IEEE, pp. 58-62, 2015.

[17] Edinger, J., Schäfer, D., Breitbach, M., \& Becker, C., "Developing Distributed Computing Applications with Tasklets," Pervasive Computing and Communications Workshops (PerCom Workshops), 2017 IEEE International Conference, pp. 94-96, 2017.

[18] Xie, J., Dan, L., Yin, L., Sun, Z., \& Xiao, Y., “An Energy-optimal Scheduling for Collaborative Execution in Mobile Cloud Computing," Computing and Communication (IEMCON), 2015 International Conference and Workshop, pp. 1-6),2015.

[19] Mohaisen, A., Tran, H., Chandra, A., \& Kim, Y., "Trustworthy Distributed Computing on Social Networks," IEEE Transactions on Services Computing, vol.7 Issue 3, pp. 333-345, 2014.

[20] Yadegaridehkordi, E., Iahad, N. A., \& Ahmad, N., Task-Technology Fit and User Adoption of Cloud-based Collaborative Learning Technologies," Computer and Information Sciences (ICCOINS), 2014 International Conference IEEE, pp. 1-6, 2014.

[21] Shahrom M. \& Aziz Z.A. 2016. Cognitively Inspired Algorithm for Imprecise Navigation. Journal of Computer Science. vol, 12 Issue 6, pp. 276-288, 2016.

[22] Rokde, G., Sun, M., Devireddy, S., Chang, S. K., \& Singhai, A., "U.S. Patent Application No. 15/401,786," Washington, DC: U.S. Patent and Trademark Office, 2018. 
[23] Shamsudin N, Abdullah H, Aziz Z.A. \& Norasiah M. Attributes contributing to Algorithmic Problem Solving Cempetencies. 5th International Conference on Software Engineering \& Computer Systems (ICSECS), 2017.

[24] Du, J., He, Y., Da, W. A. N., \& Xiao, J.,’U.S. Patent No. 9,268,590, Washington, DC: U.S. Patent and Trademark Office, 2016.

[25] Carlen, D., Heck, J., Szilagyi, M., Guis, M., Caruso, K., \& Mankin, Y. B. U.S. Patent No. 9,645,811. Washington, DC: U.S. Patent and Trademark Office. 2017. 2017.

\section{BIOGRAPHIES OF AUTHORS}

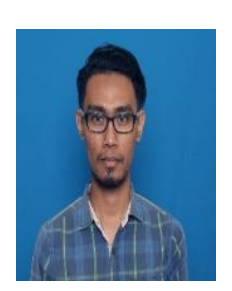

Mohd Afiq Bin Zamanhuri

Education:

- Bachelor of Computer Science (Multimedia Computing), UiTM

- Diploma in Information Technology, Cosmopoint College

Research Interest:

Multimedia, Collaborative computing

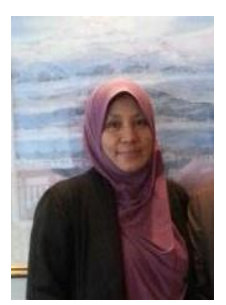

Zalilah Abd Aziz (Dr.): Senior Lecturer

Education:

- PhD in Computer Science (Artificial Intelligence) University of Nottingham

- MSc in Computer Science (Software Engineering) UPM

- BSc (Hons) Sains Komputer, ITM/UKM

Research Interest:

Software Quality, Software Engineering, Artificial Intelligence, Combinatorial Optimization

Problems, Metaheuristics, Programming

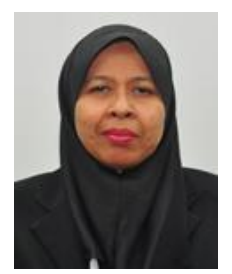

Rose Hafsah Abd Rauf (Dr.): Senior Lecturer

Education:

- $\mathrm{PhD}$, (Computer Science) Swansea University, Wales, UK 2008

- $\quad$ SSn (Sains Komp) UKM, 1997

- BSc (Comp Sc) USM, 1985

Research Interest:

Programming Language, Theory of Computation,Steganography,Computer Science Education, Digital Forensics

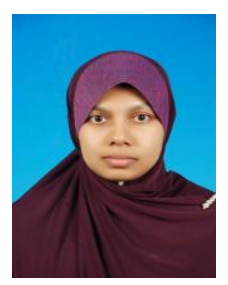

Elly Johana Johan :Senior Lecturer

Education:

- M.Sc. Information Technology (Computer Science), UKM, Malaysia (2003)

- Bachelor of Information Technology (Hons.), UiTM, Malaysia (2000)

- Diploma in Computer Science, UiTM, Malaysia (1998)

Research Interest:

Programming, Computer Science Education, Persuasive Technology

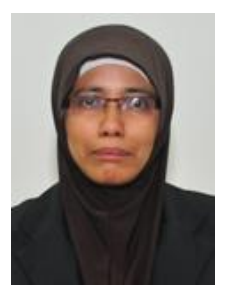

Noratikah Shamsudin

Education:

- M. Sc IT (UKM, Bangi), 2000

- B. Sc (Hons) Computer Science (UTM, KL), 1990

- Diploma in Computer Science (UTM, KL), 1988

Research Interest:

Programming, Computer Science Education, Multimedia 